\title{
Diagnóstico de Endometriosis en Medellín - Colombia
}

\author{
Dres.: Jaime Botero U.*, Aníbal Castañeda L.** \\ y Germán González E.***
}

\section{INTRODUCCION}

La ocurrencia de endometriosis en la mujer colombiana se ha considerado rara, como lo demuestran los aportes que a la literatura médica se han presentado hasta 1979 (Cuadro 1).

Amaya y otros (1) encontraron 102 casos entre 1945 y 1958 en el Hospital la Samaritana de Bogotá, el $60 \%$ de los cuales correspondía a adenomiosis y el resto a endometriosis externa. El diagnóstico preoperatorio se hizo en el $4 \%$ de las pacientes. Cardona (2) presentó en la Primera Convención Colombiana de Obstetricia y Ginecología en Bogotá en 1953, * Profesor Emérito Facultad de Medicina
Universidad de Antioquia.

** Director de Profamilia, Medellín.

*** Profesor Titular Epidemiólogo Facultad Nacional de Salud Pública, Universidad de Artioquia.

Presentado en la sesión del 30 de abril de 1986 de la Academia de Medicina de. Medellín. un estudio de 27 casos que recopiló entre 1946 y 1953 y en las tres cuartas partes de ellos se trataba de endometriósis interna. Arango (3) en Cali encontró en 55\% pacientes hospitalizadas en un servicio ginecológico, 28 casos de endometriosis. Se suponía entonces que esta era una enfermedad propia de países desarrollados en los cuales el matrimonio se presentaba tardíamente y los embarazos ocurrían después de los 25 años en una alta proporción de veces. En una revisión de las historias clínicas del Hospital Universitario San Vicente de Medellín encontramos 52 con diagnóstico de endometriosis entre 1975 y 1979 y en solamente dos casos el diagnóstico se hizo con la ayuda de la laparoscopia.

Con el uso generalizado de este procedimiento, se incrementó el hallazgo: Casas y cols. (4) encontraron entre 2.200 pacientes a quienes practicaron laparoscopia diagnóstica entre 1974 y 1979 en Profamilia en Medellín, 237 casos de endometriosis, un $10.8 \%$ del total de las pacientes estudiadas. Compárese esta cifra con el total de casos recopilados en la 


\section{CUADRO 1}

ENDOMETRIOSIS. LA FRECUENCIA DE SU HALLAZGO EN COLOMBIA DESDE 1945

\begin{tabular}{|c|c|c|c|c|}
\hline CIUDAD & AUTOR & AÑos & ND DE CASOS & NOTAS \\
\hline Cocota & AMAYA,H. & Mn-100. & 102 & nocerme LA SAMANGTANA. \\
\hline mcosun & CNNDONA,P.N. & 1046-1053 & 27 & MOSPTAL SAN VICENTE \\
\hline 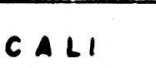 & ARANCO,J.C. & 1053 & 20 & $\begin{array}{l}\text { EWTAE } 550 \text { PACIENTES } \\
\text { HOSPITALIZADAS }\end{array}$ \\
\hline MEOELLIN & Doteng, $\mathrm{J}$ & 1075-1970 & 52 & 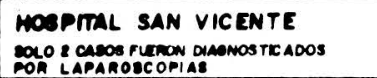 \\
\hline meocLlin & CASASg at.d & $1074-1070$ & 237 & $\begin{array}{l}\text { ENTEE } 2200 \text { I APAROSCUPIAS DIAG } \\
\text { MOSTICAS MECHAS EN PROFAMLIA }\end{array}$ \\
\hline MECLLIN & $\begin{array}{l}\text { DOTERO,J } \\
\text { CASTANEDAA. } \\
\text { CONZALEZ,O. }\end{array}$ & 1070-1984 & 310 & $\begin{array}{l}\text { LARANOSCOMAS OMONOETICAS } \\
\text { MECHAS DN PMOFAMILIA }\end{array}$ \\
\hline
\end{tabular}

literatura colombiana hasta antes del uso de la laparoscopia, que llega a 358.

En el presente estudio presentamos 506 casos diagnosticados entre enero 10. de 1979 y octubre 31 de 1985. El núme. ro de procedimientos laparoscópicos realizados en este período aparece en el Cuadro 2.

\section{CUADRO 2}

INFORME DEL NUMERO DE ACTIVIDADES ENDOSCOPICAS. PROFAMILIA, MEDELLIN, SEPTIEMBRE 1979 - OCTUBRE 1985

\begin{tabular}{|c|c|c|c|c|c|c|c|c|}
\hline 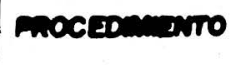 & $19 \pi$ & 1000 & 1901 & 1002 & 1983 & 1904 & $\begin{array}{l}\text { oct. \&1 } \\
10.06\end{array}$ & Totnes \\
\hline 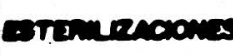 & BAI & 2003 & 3047 & 3620 & 4.052 & 4772 & 4.936 & 28.630 \\
\hline $\begin{array}{l}\text { Dx } \\
\text { LAMnOScopias }\end{array}$ & 140 & 1.540 & 2044 & 1.449 & 3.335 & 3604 & 3.007 & 16.472 \\
\hline & & & & & & TOT & & 43.102 \\
\hline
\end{tabular}




\section{CUADRO 3}

\section{ENDOMETRIOSIS-INDICACION PARA LAPAROSCOPIA. PROFAMILIA, MEDELLIN 1985}

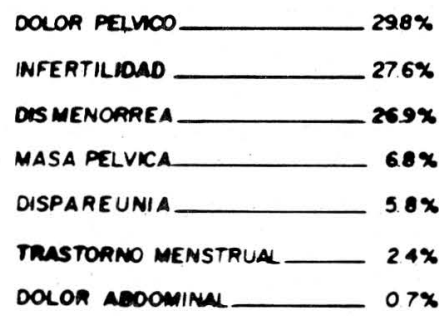

\section{METODOLOGIA}

La población seleccionada está constituida por las pacientes atendidas en Profamilia entre las fechas mencionadas arriba, a quienes se hizo el diagnóstico de endometriósis mediante el procedimiento de una laparoscopia. Los casos se tomaron de un tarjetero existente en la institución que contiene la identificación de las pacientes $y$ el diagnóstico que se ha hecho con el procedimiento. La información se recolectó de la historia clínica mediante un formulario que se elaboró y que fue probado previamente con un número significativo de historias. Los datos fueron luego pasados para su procesamiento a un microcomputador personal NEC 8801A, utilizando el programa Dbase II (5).

\section{RESULTADOS}

El gráfico 1 muestra la frecuencia con la cual se hizo el diagnóstico de endometriósis en los casos de laparoscopias diagnósticas, observándose un incremento brusco desde 1982, que significa posiblemente la prevalencia de la enfermedad y luego un descenso moderado que en el futuro puede indicarnos la verdadera incidencia de la misma en nuestra población. Un hallazgo similar se presenta en el gráfico 2 que muestra los casos diagnosticados en las laparoscopias que se efectuaron con fines de esterilización.

$$
\text { GRAFICO } 1
$$

FRECUENCIA DEL HALLAZGO DE ENDOMETRIOSIS DE ACUERDO CON EL NUMERO DE LAPAROSCOPIAS DIAGNOSTICAS, MEDELLIN, 1984

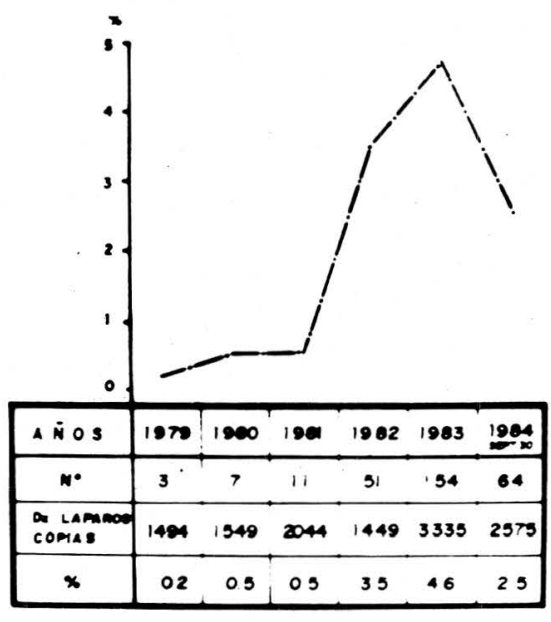

GRAFICO 2

FRECUENCIA DEL HALLAZGO DE ENDOMETRIOSIS DE ACUERDO AL NUMERO DE LAPAROSCOPIAS PARA ESTERILIZACION, MEDELLIN, 1984

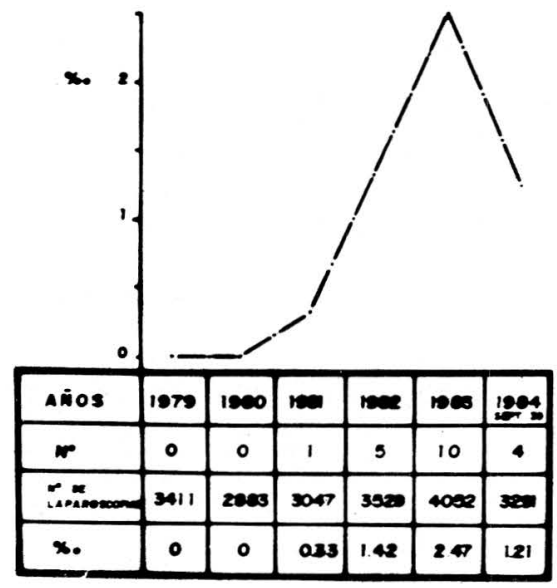


El gráfico 3 muestra cómo el hallazgo de la enfermedad es más frecuente en el grupo de 25 a 34 años, en cualquiera de los tres estadios que se considere. El gráfico 4 compara la distribución de la población femenina en el Valle de Aburrá, donde está situada la ciudad de Medellín, con la distribución de los casos de endometriósis, observándose también que en el grupo de 25 a 29 y de 30 a 34 años y un poco menos en el siguiente, la proporción de la enfermedad es mayor que la de la población.

En la tabla 3 observamos las principales indicaciones para la laparoscopia diagnóstica. Llama la atención que el trastorno menstrual ocupa un lugar secundario con un escaso número de pacientes y que el primer lugar lo ocupa el dolor.

En el gráfico 5 obscrvamos los diferentes tipos de tratamiento en el estadio I.
GRAFICO 3

ENDOMETRIOSIS. GRADO CLINICO (AFS) DE ACUERDO A LOS GRUPOS DE EDAD. PROFAMILIA, MEDELLIN,

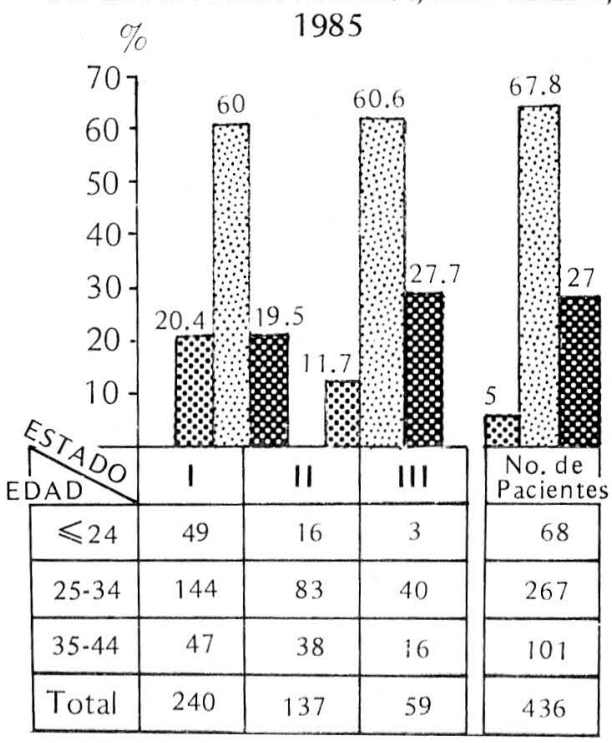

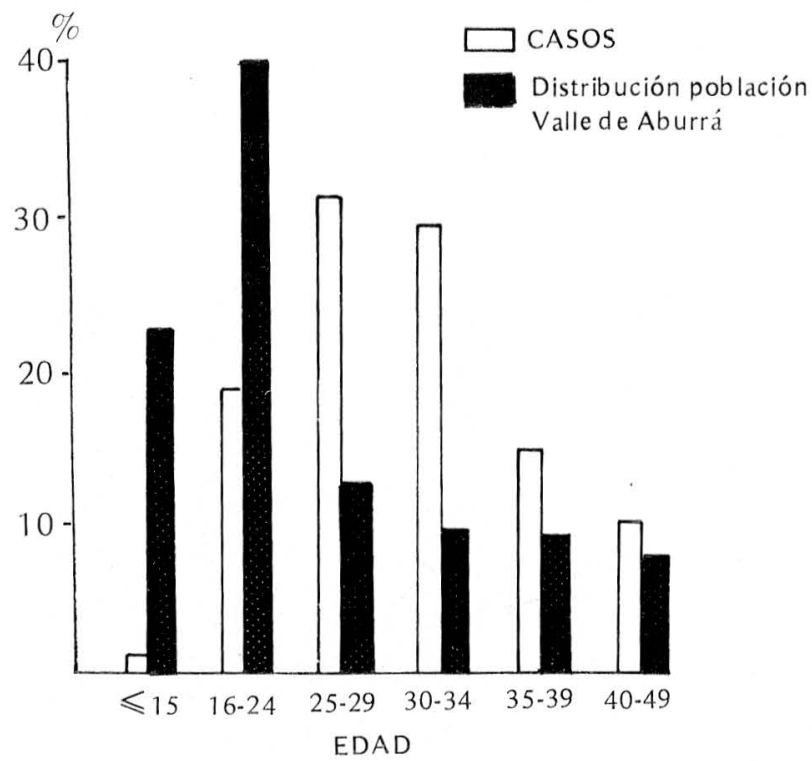

GRAFICO 4

DISTRIBUCION POR

EDADES DE LOS CASOS

DE ENDOMETRIOSIS

HALLADOS EN

LAPAROSCOPIAS

DIAGNOSTICAS

COMPARADA CON! LA

POBLACION FEMENINA

DEL VALLE

DE ABURRA.

MEDELLIN, 1984 
GRAFICO 5

ENDOMETRIOSIS - TIPOS DE TRATAMIENTO EN EL ESTADIO I. PROFAMILIA, MEDELLIN, 1985

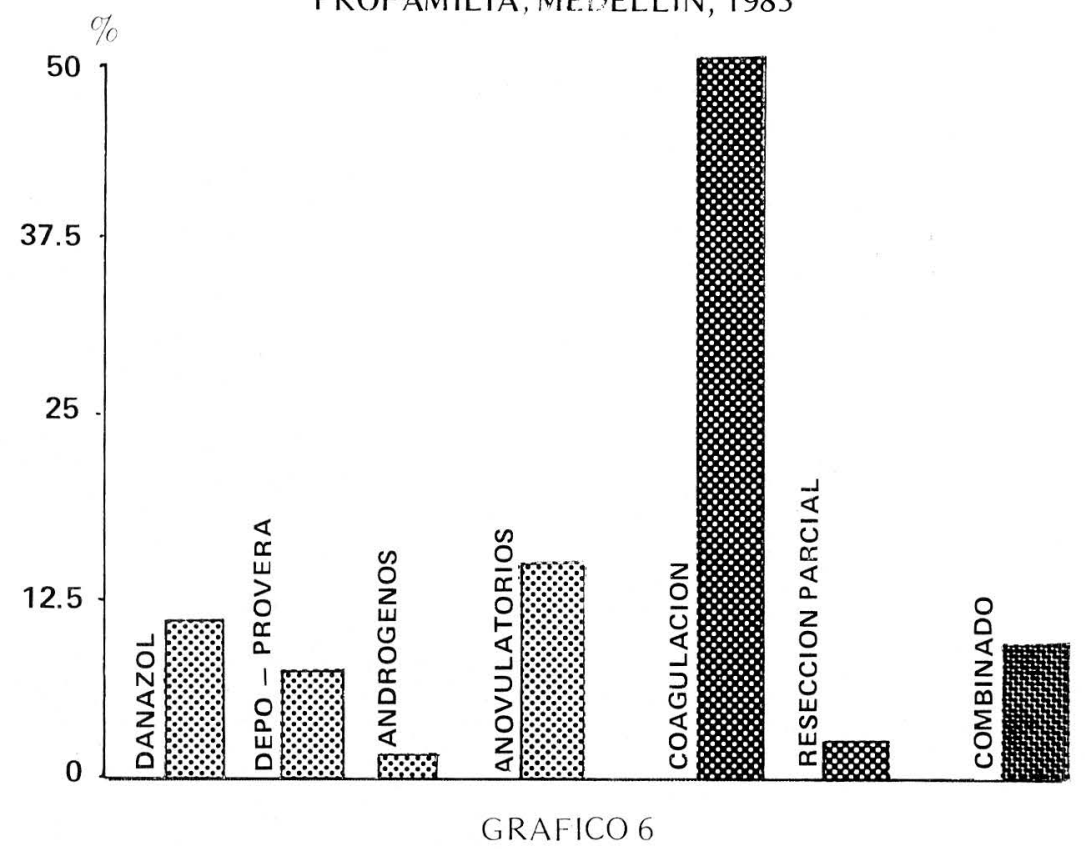

ENDOMETRIOSIS - TIPOS DE TRATAMIENTO EN EL ESTADIO II. PROFAMILIA, MÉDELLIN, 1985

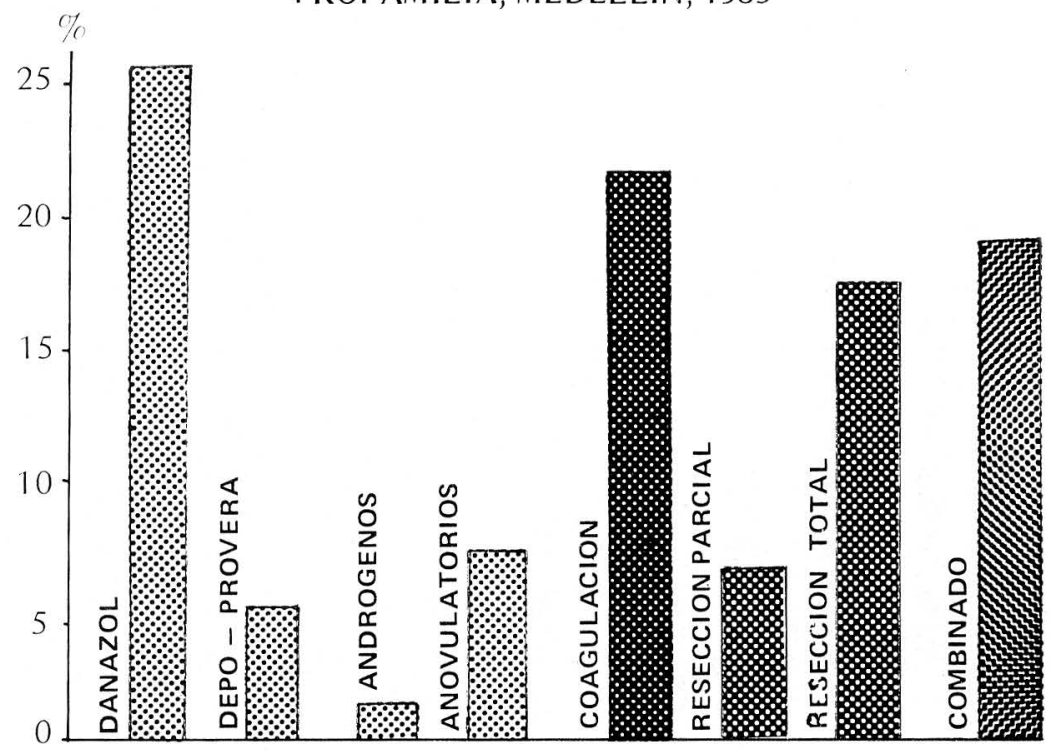


GRAFICO 7

ENDOMETRIOSIS - TIPOS DE TRATAMIENTO EN EL ESTADIO III.

PROFAMILIA, MEDELLIN, 1985

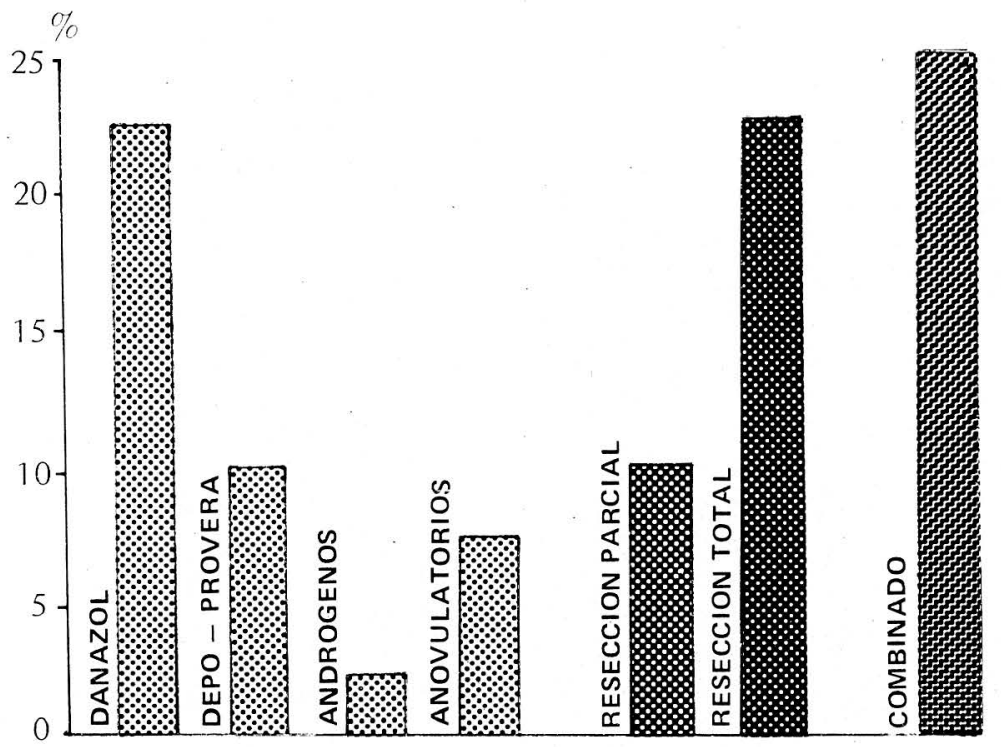

GRAFICO 8

MODALIDAD TERAPEUTICA Y TIPO DE RESPUESTA AL TRATAMIENTO DE ENDOMETRIOSIS. MEDELLIN, 1985

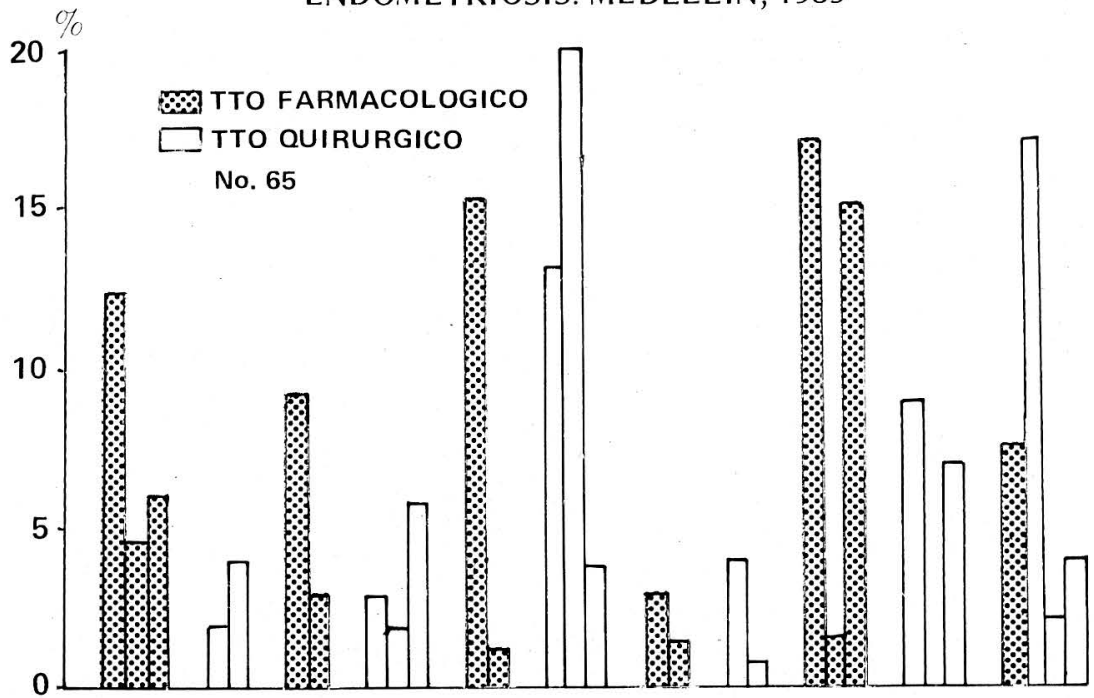

RESPUESTA LEVE MODERADO CURACION EMBARAZO NO MEJORIA DESCONOCIDO 
Hay una gran predilección por la coagulación y un uso restringido de los métodos farmacológicos, entre los cuales no aparecen los progestágenos sintéticos. En el estadio 11 (gr. 6) en cambio se recurrió al danazol en una proporción importante de casos, de nuevo a la coagulación de focos en otro porcentaje importante $y$ en menos proporción a la resección total y a los tratamientos combinados. No se discrimina en este momento cuántos casos se trataron por infertilidad y cuántos por sintomas incapacitantes. Sólo queremos ver la predilección por las diferentes formas terapéuticas en los distintos estadios. En el III se usa el danazol, la resección total y el tratamiento mixto en una proporción igual de casos, como se observa en el gráfico 7.

Solamente a título informativo presentamos el gráfico 8, que muestra las diferentes modalidades terapéuticas y el tipo de respuesta a las mismas. Ahí se observa toda una gama de resultados, sin que pueda sacarse una conclusión valedera debido al escaso número de pacientes en cada grupo, al no empleo de la técnica aleatoria para la escogencia de las mismas y a la ausencia de grupos de control.

\section{DISCUSION}

El grupo de pacientes que hemos presentado en este estudio representa muy seguramente la mayoría de casos de endometriosis que ocurren en la comunidad que habita el área metropolitana de Mcdellín. Las principales instituciones hospitalarias de esta zona envían a Profamilia los casos en que es preciso practicar laparoscopia diagnóstica para llegar a un diagnóstico etiológico. El procedimiento siempre lo realiza el mismo grupo médico, con entrenamiento muy completo y la clasificación se hace siguiendo las pautas de la American Fertility Society (6).

El aumento en la frecuencia del diagnóstico que se ha observado en los últimos años puede atribuirse más a una mayor asiduidad en la búsqueda de la enfermedad, que a un incremento en la ocurencia de la misma. El descenso que ocurre más adelante (ver tabla 3), puede empezar a indicar la verdadera incidencia de la endometriosis en esta población.

Parece existir un factor de riesgo que se manifiesta en forma constante en los grupos de edad que se forman a partir de los 25 años. Puede ser de origen endógeno (¿hormonal?) o exógeno, (exámenes vaginales, presencia de dispositivo intrauterino, ingestión de hormonas, etc.). Algunas consideraciones pueden hacerse en relación con el tratamiento en general de la endometriosis. Los que se han usado varían mucho en sus resultados scgún los motivos para los cuales se hacen: alivio do los síntomas o mejoría de la fertilidad $y$ según los autores que los han estudiado. Seguramente a ello contribuye también el rigor metodológico que se aplique en la investigación. Los porcentajes que se dan para mejoría de los síntomas van desde el $30 \%$ (7) al $94 \%$ (8) con cirugía conservadora y son del $40 \%$ (9) con el uso de danazol.

Para el tratamiento de la infertilidad los resultados varían desde un $38 \%$ de tasa de embarazos (10) hasta un $83 \%$ (11) y aún se han presentado unos tan bajos como los de Butler y otros (12) que dan una tasa de embarazos viables de sólo un $20 \%$ con el uso de danazol.

En nuestro grupo de pacientes parece que el tratamiento quirúrgico fuera superior al farmacológico para la curación de los síntomas. 


\section{CONCLUSION}

Hemos encontrado una vez más que la endometriosis no es tan rara en esta población y puede representar una proporción importante entre las enfermedades que causan sintomatología pélvica y producen infertilidad, determinando a su vez incapacidad laboral. Creemos que con este estudio descriptivo se plantea la necesidad de hacer otros adicionales siguiendo dos orientaciones principales:

a. Buscar la asociación entre la enfermedad y un posible factor de riesgo: drogas, dispositivos intrauterinos, exámenes pélvicos, comportamiento sexual, etc., mediante un estudio de casos y controles.

b. Comparar distintos tratamientos médicos y quirúrgicos, para ver las ventajas del uno sobre el otro, no sólo en relación con la mejoría de los síntomas sino de la fertilidad y también en relación con el costo-beneficio de cada uno de ellos. Somos conscientes de que deben usarse metodologías adecuadas a las peculiaridades que presentan este tipo de pacientes (13).

\section{RESUMEN}

Hasta 1979 el diagnóstico de endometriosis en Colombia se hacía muy rara. mente. Con la introducción de la laparoscopia se empezó a encontrar la enfermcdad con una mayor frecuencia. En este estudio se encontró con una frecuencia variable pero la tendencia de la curva en los distintos años desde 1979 hasta sep- tiembre de 1984 parece indicar que se encuentra en el $2.5 \%$ de las laparoscopias diagnósticas $y$ en el 1.21 por mil de las tubectomías laparoscópicas que se realizan en Profamilia, en Medellín, Colombia.

El riesgo de endometriosis parece aumentar con la edad de las mujeres y parece ser mayor para dos grupos de edad: el de 25-29 años en donde se presentan el $30.3 \%$ de los casos mientras que la población femenina de esa edad sólo representa el $12.7 \%$ de la que compone el valle de Aburrá, donde está situada Medellín $y$ el de 30-34 años en donde ocurre el $28.2 \%$ de los casos mientras que la población femenina de esa edad es sólo el 9.7\% de la que habita en el área mencionada. El mismo factor de riesgo parece estar actuando a medida que aumenta la edad de la paciente, determinando formas más severas en los grupos de edades mayores, pero sólo en una proporción ligeramente más alta.

El dolor, que se manifiesta por disemenorrea en un $26.9 \%$ de los casos y por dolor pélvico en un $29.8 \%$, representa la mitad de las indicaciones para laparoscopia diagnóstica en la cual se encontró endometriosis.

El aparente mejor resultado del tratamiento quirúrgico sobre el conseguido con el uso de drogas, merece un estudio mejor controlado en el futuro.

Igualmente se considera oportuno el estudio de modalidades terapéuticas que estén más al alcance de nuestra población desde el punto de vista del costo y que tengan una equivalente eficacia curativa. 


\section{ABSTRACT}

Until the introduction of the laparoscope for diagnostic purposes, endometriosis was diagnosed very infrequently in Colombia. Only 358 cases were reported in the Colombian medical literature until Casas et al (1979) reported 237 cases among 2.200 patients undergoing diagnostic laparoscopy between 1974 and 1979 , for an incidence of $10.8 \%$ in the patients studied.

An analysis of 310 cases diagnosed between January 1979 and September

\section{BIBLIOGRAFIA}

1. AMAYA H., CORREA H., ESPINOSA A., RUEDA R., VERGARA R., VILLARREAL J., Endometriosis: consideraciones sobre su incidencia en Bogotá. Rev. Colombiana Obstetricia y Ginecología 11 (No. 3) 351-358, 1960.

2. CARDONA P. N., Endometriósis. Rev. Colombiana Obstetricia y Ginecología 5 (No. 5): 133, 1954.

3. ARANGO J. C., Individualidad de la endometriosis estromatógena. Rev. Colombiana Obstetricia y Ginecología. Memorias de la II Convención Colombiana de Obstetricia y Ginecología (Ed. Especial). pág. 256, 1955.

4. CASAS G., CASTAÑEDA A., RIOS B., RODRIGUEZ L., TOBON G., Informe sobre la tabulación de 2.200 historias de pacientes de Profamilia de Medellín, a quienes se les practicó laparoscopia diagnóstica. 1979. Informe mimeografiado. Presentado en XIII Congreso Colombiano de Obstetricia y Ginecología Bogotá, Diciembre 1979.
1984 revealed that the risk of endometriosis increases with age and is greatest in patients aged 25-29 and 30-34. Of the total cases, $30.3 \%$ occurred in the former age group whereas that age group constitutes only $12.7 \%$ of Medellin women. In women $30-34$ years old, $28.2 \%$ of the cases occurred while that age group constitutes only $9.7 \%$ of Medellin women. Dysmenorrhea and generalized pelvic pain were the indications for diagnostic laparoscopy in $50 \%$ of the cases in which a diagnosis of endometriosis was made.

5. ASHTON-TATE, dBase 11. Assembly Language Relational Database Management System. Versión 2.3B 22 Febrero 1982.

6. THE AMERICAN FERTILITY SOCIETY: Classification of Endometriosis. Fertil Steril 32(6):633-634 (Dec.), 1979.

7. SPANGLER D. B., JONES G. S., JONES H. W. Jr., Infertility due to endometriosis. Am J. Obstet Gynecol 109: 850, 1971.

8. McCOY J. B., BRADFORD W. Z., Surgical treatment of endometriosis with conservation of reproductive potential. Am J. Obstet Gynecol 87: 394, 1963.

9. FRIEDLANDER R. L., The treatment of endometriosis with danazol J. Reprod. Med. 10: 197, 1973.

10. SEIBEL M. M. BERGER M. J., WEINSTEIN F., TAYMOR M. L., The effectiveness of danazol on subsequent fertility 
in minimal endometriosis. Fertil Steril (Abstr) 37: 310, 1982.

11. DMOWSKI W. P., COHEN M. R., Antigonadotropin (danazol) in the treatment of endometriosis: evaluation of posttreatment fertility and three-year followup data. Am J. Obstet Gynecol 130: 41, 1978.
12. BUTLER L., WILSON E., BELISLE S., GIBSON M., ALBRECHT B., SCHIFF I., STILLMAN R.: Collaborative study of pregnancy rates following danazol therapy of stage I endometriosis Fertil Steril 41: 373, 1984.

13. LERIDON H., SPIRA A., Problems in measuring the effectiveness of infertility therapy. Fertil Steril 41: 580, 1984. 